\title{
Patents, programs and proteins
}

\section{Personalized search, personalized plasmids.}

\section{Science IP}

Chemical Abstracts Service

www.sciencelP.org

An on-demand search service

Science IP is a personalized search service for information professionals, attorneys and others involved in patenting and research and development. Information obtained from this source is suitable for supporting decisions on prior art/patentability for the prosecution of new patents, freedom-to-practice/operate for new and existing technologies, and patent validity for infringement litigation. The search draws upon CAS scientists with knowledge of specialized areas, including nucleic and amino acid sequences, polymers, molecular biology and genetics and pharmaceuticals.

\section{DNASIS MAX 2.0}

Hitachi Software Engineering

www.hitachisoft-bio.com

\section{Sequence analysis software upgrade}

The DNASIS MAX 2.0 software package for Windows includes a wide range of standard sequencing functions. It features automated, customizable sequence analysis, allowing users to create and save workflows for specific requirements. Enhancements to this version include Contig Manager, a DNA sequence assembly tool for sequencing projects, and Plasmid Map Editor, which can be used to construct the plasmid map supported by various objects such as lines, arrows, labels or texts.

\section{Knockout RNAi System}

BD Biosciences/Clontech www.clontech.com Multiple delivery options for gene silencing

The Knockout RNAi system from BD Biosciences Clontech provides retroviral and adenoviral delivery formats that generate siRNA molecules for effective gene silencing in many cell types. The RNAi-ready pSIREN vectors are ligation-ready plasmids that allow users to choose plasmid or viral delivery. The system overcomes difficulties associated with plasmid-based vectors - namely, the limited ability to deliver siRNA to various cell types. To prepare a construct, users select target sequences within the coding region of the gene of interest and synthesize a doublestranded hairpin oligo for each target sequence. The oligo is then ligated into a pSIREN vector to express the siRNA.

\section{p3 microplate \\ Porvair Sciences} Double feature

The p3 microplate has a dual filter design to prevent sample breakthrough and waste associated with leakages from traditional protein precipitation microplates. Hydrophobic/ oleophobic filters retain the entire acetonitrile/sample precipitation mix, allowing complete mixing without loss or leakage. A pre-filter retains large flocculent particles, eliminating 'plugging' problems, while the final filter traps residual protein particles. The large pore size improves flow rate. The $\mathrm{p} 3$ processes 96 samples per batch and will integrate with automated systems.

\section{Plasmid Prep Service}

Qiagen www.qiagen.com/plasmidprepservice Promptpreps

Qiagen's Plasmid Prep Service provides ultrapure plasmid DNA in milligram to gram amounts for research and pre-clinical applications. The plasmid DNA is suitable for gene therapy or genetic vaccination projects and for transfection into sensitive eukaryotic cell lines. The company's plasmid purification specialists can help with individual needs, and a range of extra options are available, including lowand ultralow-endotoxin grades, bio-burden, host protein and endotoxin assays and restriction analysis. A certificate of analysis is delivered with each plasmid DNA prep. Preps can be ordered online for rapid turnaround.

These notes are compiled in the Nature office from information provided by the manufacturers. 